\title{
Non-alcoholic fatty liver disease and cardiovascular disease: assessing the evidence for causality
}

\author{
Martijn C. G. J. Brouwers ${ }^{1,2}$ (D) Nynke Simons ${ }^{1,2,3} \cdot$ Coen D. A. Stehouwer $^{2,4} \cdot$ Aaron Isaacs $^{2,5,6}$
}

Received: 6 June 2019 / Accepted: 29 July 2019/Published online: 11 November 2019

(C) The Author(s) 2019

\begin{abstract}
Non-alcoholic fatty liver disease (NAFLD) is highly prevalent among individuals with type 2 diabetes. Although epidemiological studies have shown that NAFLD is associated with cardiovascular disease (CVD), it remains unknown whether NAFLD is an active contributor or an innocent bystander. Plasma lipids, low-grade inflammation, impaired fibrinolysis and hepatokines are potential mediators of the relationship between NAFLD and CVD. The Mendelian randomisation approach can help to make causal inferences. Studies that used common variants in PNPLA3, TM6SF2 and GCKR as instruments to investigate the relationship between NAFLD and coronary artery disease (CAD) have reported contrasting results. Variants in PNPLA3 and $T M 6 S F 2$ were found to protect against CAD, whereas variants in GCKR were positively associated with CAD. Since all three genes have been associated with non-alcoholic steatohepatitis, the second stage of NAFLD, the question of whether low-grade inflammation is an important mediator of the relationship between NAFLD and CAD arises. In contrast, the differential effects of these genes on plasma lipids (i.e. lipid-lowering for PNPLA3 and TM6SF2, and lipid-raising for GCKR) strongly suggest that plasma lipids account for their differential effects on CAD risk. This concept has recently been confirmed in an extended set of 12 NAFLD susceptibility genes. From these studies it appears that plasma lipids are an important mediator between NAFLD and CVD risk. These findings have important clinical implications, particularly for the design of anti-NAFLD drugs that also affect lipid metabolism.
\end{abstract}

Keywords Cardiovascular disease $\cdot$ Coronary artery disease $\cdot$ GCKR $\cdot$ Mendelian randomisation $\cdot$ Non-alcoholic fatty liver disease $\cdot$ Non-alcoholic steatohepatitis $\cdot P N P L A 3 \cdot$ Review $\cdot$ TM6SF2

$\begin{array}{ll}\text { Abbreviations } \\ \text { CAD } & \text { Coronary artery disease } \\ \text { CETP } & \text { Cholesteryl ester transfer protein } \\ \text { CRP } & \text { C-reactive protein } \\ \text { CVD } & \text { Cardiovascular disease }\end{array}$

Electronic supplementary material The online version of this article (https://doi.org/10.1007/s00125-019-05024-3) contains a slideset of the figures for download, which is available to authorised users.

Martijn C. G. J. Brouwers

mcgj.brouwers@mumc.nl

1 Division of Endocrinology and Metabolic Disease, Department of Internal Medicine, Maastricht University Medical Centre, P. Debyelaan 25, 6229 HX Maastricht, the Netherlands

2 Cardiovascular Research Institute Maastricht (CARIM), Maastricht University, Maastricht, the Netherlands
GKRP Glucokinase regulatory protein

MR Mendelian randomisation

NAFLD Non-alcoholic fatty liver disease

NASH Non-alcoholic steatohepatitis

PAI-1 Plasminogen activator inhibitor type 1
3 Laboratory for Metabolism and Vascular Medicine, Division of General Internal Medicine, Department of Internal Medicine, Maastricht University Medical Centre, Maastricht, the Netherlands

4 Division of General Internal Medicine, Department of Internal Medicine, Maastricht University Medical Centre, Maastricht, the Netherlands

5 Maastricht Centre for Systems Biology (MaCSBio), Maastricht University, Maastricht, the Netherlands

6 Department of Biochemistry, Maastricht University, Maastricht, the Netherlands 
PNPLA3 Patatin-like phospholipase domain-containing protein 3

TM6SF2 Transmembrane 6 superfamily 2

\section{Introduction}

Non-alcoholic fatty liver disease (NAFLD) is a frequently encountered phenomenon in type 2 diabetes. It is a histological spectrum consisting of hepatic fat accumulation ('simple steatosis'), non-alcoholic steatohepatitis (NASH), fibrosis and cirrhosis, occurring in the absence of excessive alcohol intake. It has been estimated that the global prevalence of NAFLD is $25 \%$ and that $23 \%$ of affected individuals have type 2 diabetes [1].

The pathogenesis of NAFLD involves a complex interaction between genetic and environmental factors. The accumulation of hepatic fat is the consequence of an imbalance between the influx of fat (i.e. fatty acids from adipose tissue or diet and de novo lipogenesis from glucose) and the efflux of fat (i.e. $\beta$-oxidation and synthesis of VLDL). Stable isotope studies have demonstrated that an increased fatty acid flux and de novo lipogenesis are the two principal processes contributing to hepatic fat accumulation in individuals with NAFLD [2]. Although hepatic fat accumulation is a prerequisite for the development of NASH, not all individuals with simple steatosis proceed to this stage. Lipotoxicity, the key driver behind the development of NASH, is determined by the following factors: (1) both the quantity and type of lipids that accumulate; and (2) the ability of the liver to defend against lipotoxicity [3].

Although NAFLD is a risk factor for end-stage liver disease and hepatocellular carcinoma (it is projected to be the principal cause of liver transplantation by 2025 [4]), individuals with NAFLD mostly die from cardiovascular disease (CVD) [5]. A previous meta-analysis demonstrated that the risk of developing a fatal and/or non-fatal CVD event is $64 \%$ higher in individuals with vs without NAFLD [6]. Of note, in the majority of the studies that were included in this meta-analysis, the diagnosis of NAFLD was established by either ultrasound or computed tomography [6], which are only capable of diagnosing simple steatosis, not advanced stages of NAFLD. In contrast, one of the few studies that included histologically confirmed NAFLD, and showed that advanced liver fibrosis specifically accounted for the greater CVD risk, was limited by a relatively small sample size and a selected population that underwent a liver biopsy [7].

There is an ongoing discussion on whether NAFLD is truly an active contributor or an innocent bystander in the development of CVD, as recently reviewed [8]. It is often presumed that the association between NAFLD and CVD is confounded by NAFLD-related factors (i.e. the association of NAFLD with other factors related to the metabolic syndrome, such as dyslipidaemia, hypertension and type 2 diabetes) and this could theoretically explain the association between NAFLD and CVD [8-10]. Of interest, NAFLD was found to be the strongest determinant of increased intima-media thickness, independent of the potential confounding effects of age, sex, visceral fat mass, state of hyperglycaemia, insulin resistance and insulin secretion, in individuals with impaired fasting glucose and/or impaired glucose tolerance [11]. It should, however, be noted that some of these factors are not necessarily confounders but instead could act as mediators of the relationship between NAFLD and CVD (Fig. 1). Since NAFLD and CVD are biologically distant traits, they must be linked through mediating factors. We will argue that plasma lipids act as an important mediator between NAFLD and CVD.

Therefore, the present review has several aims: (1) to elaborate on potential mediators of the relationship between NAFLD and CVD; (2) to provide experimental evidence for a causal relationship between NAFLD and CVD; and (3) to discuss clinical implications.

\section{Potential mediators of the association between NAFLD and CVD}

The pathogenesis of an atherosclerotic plaque, the pathological lesion that is responsible for a cardiovascular event, consists of several stages (Fig. 2), as reviewed in detail elsewhere [12]. NAFLD could theoretically contribute to all of these stages.

NAFLD and dyslipidaemia Individuals with NAFLD have a typical plasma lipid pattern characterised by elevated plasma triacylglycerols, low HDL-cholesterol and a high number of circulating small-dense LDL particles [13]. Stable isotope studies have shown that NAFLD is associated with (insulin-resistant) overproduction of triacylglycerol-rich VLDL particles [14]. In plasma, triacylglycerols from VLDL particles are exchanged for cholesteryl esters from LDL and HDL particles, a process mediated by cholesteryl ester transfer protein (CETP). Once these triacylglycerols have been hydrolysed by hepatic lipase, both LDL and HDL particles become small and cholesterol-depleted [15].

The Mendelian randomisation (MR) approach can help to make causal inferences, as summarised in Text box 1 and comprehensively described elsewhere [16, 17]. Previous MR studies have demonstrated that plasma triacylglycerols are causal in the pathogenesis of coronary artery disease (CAD) [18], whereas low plasma HDL-cholesterol levels do not necessarily play an active role in the pathogenesis of CVD [19]. Experimental studies have shown that small-dense LDL particles are highly atherogenic [20]. 


\section{Mendelian randomisation}

General principles The human genome contains four to five million common gene variants, called SNPs, which contribute to phenotypic variation. Since these SNPs are randomly distributed among the future haploid cells during meiosis, they can be used as instruments to make causal inferences, similar to a randomised controlled trial. For instance, if we are interested in whether CRP is causally involved in the pathogenesis of CAD, we can study variants in the CRP gene that are known to affect circulating CRP levels, in relation to CAD.

Assumptions For a genetic variant to be used as a suitable instrument in an MR study, it should meet the following criteria: (1) be associated with the risk factor of interest (in this case NAFLD); (2) not be associated with any confounding factor; and (3) not be associated with the outcome (in this case CAD) through other biological pathways. Violation of the latter two principles can occur under the following conditions: (1) pleiotropy (i.e. the genetic variant affects multiple risk factors); (2) linkage disequilibrium between instrumental SNPs (i.e. the genetic variant co-segregates with neighbouring variants that also affect the outcome of interest); and (3) population stratification (i.e. the population under investigation consists of multiple [ethnic] subpopulations with different allelic frequencies) [16, 17].

Limitations The risk of pleiotropy increases when the risk factor of interest is a complex, non-protein phenotype, such as NAFLD, since there is no gene that specifically encodes NAFLD. It is important to distinguish between horizontal and vertical pleiotropy. Horizontal pleiotropy violates the MR assumption (confounding), whereas vertical pleiotropy is downstream in the pathway of interest (mediation) and therefore does not violate the MR assumption $[16,17]$.

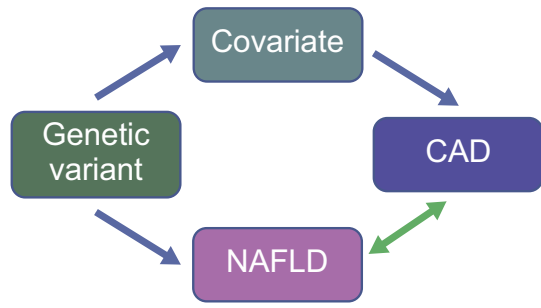

Horizontal pleiotropy: the genetic variant affects both the covariate and NAFLD. The covariate is only causally related to CAD (blue arrow). The relationship between NAFLD and $\mathrm{CAD}$ is only associative (green bidirectional arrow)

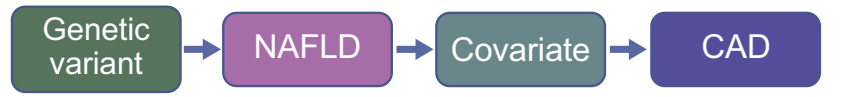

Vertical pleiotropy: the genetic variant affects both the covariate and NAFLD. The covariate is a direct effect of NAFLD and causally related to $\mathrm{CAD}$ (blue arrow)

Statistical power is another threat to the MR approach. Since common gene variants generally have small effect sizes, large cohorts are required to ensure sufficient statistical power. Both the risk factor and outcome of interest are often not assessed in the same large cohort (as is the case for NAFLD and CAD).

These limitations can be overcome by combining (summarised) data from different cohorts (one containing the genetic variant and the risk factor, the other the genetic variant and the outcome of interest) and by using multiple genetic variants. Horizontal pleiotropy may balance out when multiple genetic variants are used as an instrument. This can be formally tested (e.g. by an MR-Egger test) $[16,17]$.

NAFLD and low-grade inflammation NASH could theoretically create a systemic, low-grade inflammatory environment that promotes the pathogenesis of atherosclerosis by secreting cytokines and acute-phase proteins [8,9]. Although C-reactive protein (CRP) is a liver-specific protein that has been associated with both NASH and CVD [21, 22], MR studies have revealed that CRP is merely a biomarker, not a mediator, of CVD risk [22].

The clinical relevance of low-grade inflammation in the pathogenesis of CVD has recently been demonstrated unequivocally by the Canakinumab Anti-inflammatory Thrombosis Outcome
Study (CANTOS), in which treatment with canakinumab, a monoclonal antibody targeting IL- $1 \beta$, reduced recurrent CVD events independent of lipid lowering [23]. Of interest, IL-1 $\beta$ also plays an active role in the pathogenesis of NASH in different animal models [24]. It is not known, however, whether IL$1 \beta$ mediates the association between NASH and CVD.

NAFLD and thrombosis The rupture of a vulnerable atherosclerotic lesion and subsequent thrombosis is clinically manifested as an acute ischaemic event. Plasminogen activator 

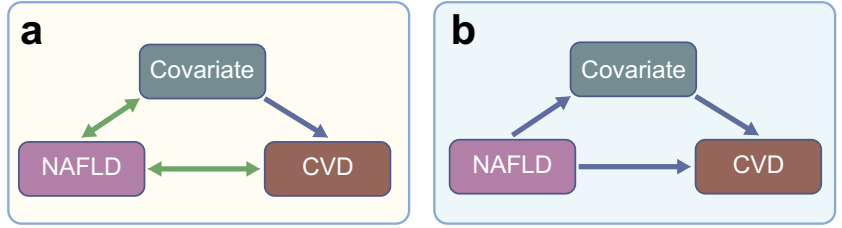

Fig. 1 Confounding and mediation. The relationship between NAFLD and CVD, as reported in observational studies, can be explained by confounding or mediation. (a) In confounding, there is a covariate that is (non-causally) associated with NAFLD (green bidirectional arrow) and causally related to CVD (blue arrow), which explains the non-causal association between NAFLD and CVD. In other words, NAFLD is an innocent bystander. (b) In mediation, there is a covariate that is the direct result of NAFLD and causally relates to CVD. In other words, NAFLD is an active contributor to CVD risk, mediated by the covariate. This figure is available as part of a downloadable slideset

inhibitor type 1 (PAI-1) is an important inhibitor of the fibrinolytic system and, hence, the resolution of a thrombus. Previous studies have shown that PAI-1 levels are elevated in individuals with NAFLD and that the liver is a principal determinant of plasma PAI-1 levels [25]. MR studies have demonstrated that elevated PAI-1 levels have a causal effect on CAD [26].

Other mediators NAFLD has been associated with insulinresistant, endogenous glucose production and, consequently, incident type 2 diabetes [27,28]. MR studies have shown that plasma glucose is causally related to CAD [29], possibly by promoting monocyte/macrophage adhesion to the endothelium, chemokine secretion by vascular smooth muscle cells, and expression of an inflammatory phenotype in macrophages [30]. More recently, the so-called hepatokines have emerged as potential mediators of cardiometabolic complications in NAFLD [31]. Of these, fetuin A has been associated with CVD [32]. Causality was inferred by one MR study [33] but this was not replicated in an MR analysis that included prospective studies [34]. Experimental studies have shown that fetuin A induces low-grade inflammation in concert with fatty acids [35].

\section{Assessing the evidence for a causal relationship between NAFLD and CVD}

Intervention studies Currently, there are two drugs that have been shown to affect both NAFLD and CVD (i.e. pioglitazone and liraglutide). Pioglitazone is highly effective in the treatment of biopsy-proven NASH (number needed to treat to resolve NASH after 18 months' treatment: 3) [36]. The Prospective Pioglitazone Clinical Trial in Macrovascular

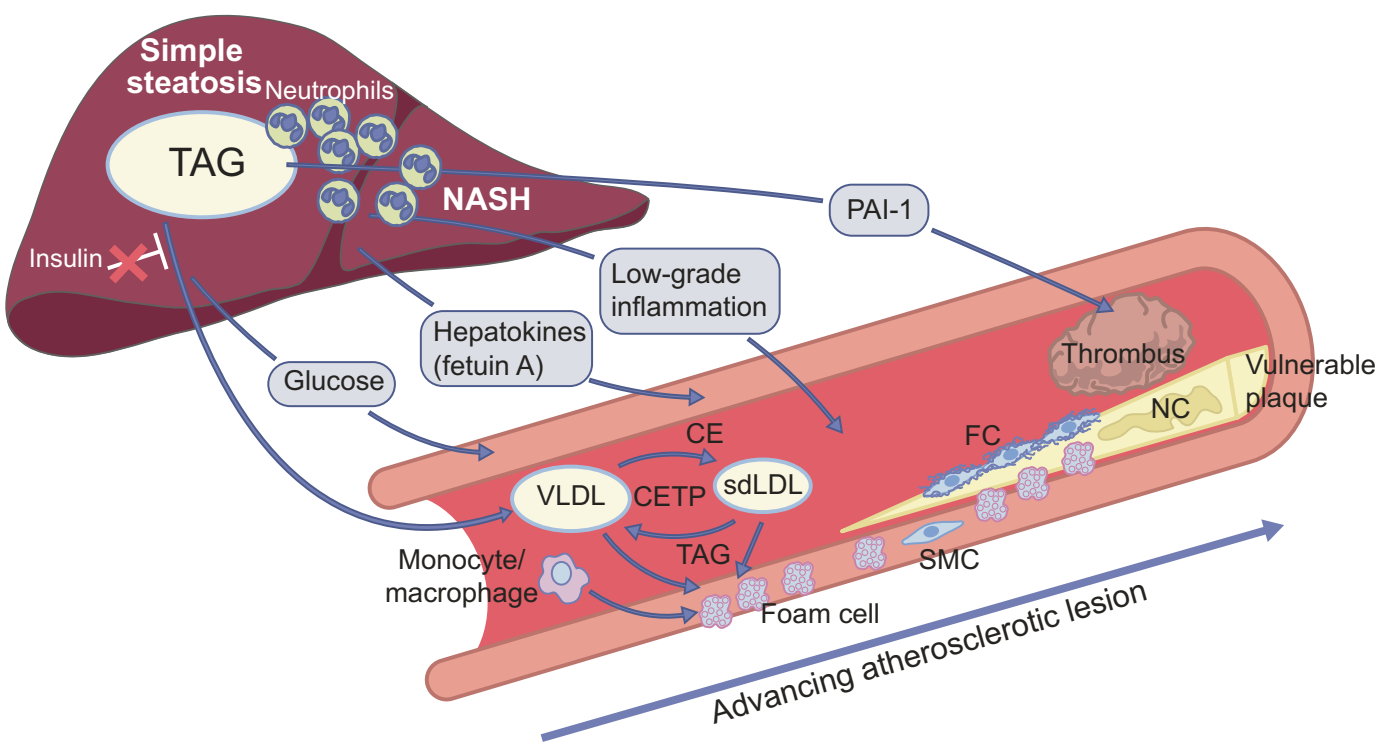

Fig. 2 Biologically plausible mechanisms that link NAFLD to the pathogenesis of an atherosclerotic lesion. The combination of endothelial dysfunction and lipoprotein retention in the arterial wall triggers a lowgrade inflammatory response, which results in the accumulation of lipidloaded, monocyte-derived macrophages ('foam cells') and the proliferation of smooth muscle cells (SMCs). This newly formed lesion is covered by a thin fibrous cap (FC). The so-called 'vulnerable plaque' consists of a necrotic core (NC) and a fibrous cap, which makes it prone to rupture. The subsequent exposure of pro-thrombotic, necrotic material to the blood stream results in acute thrombosis and occlusion of the artery. Simple steatosis (shown on the left side of the liver) and insulin resistance drive overproduction of triacylglycerol-rich VLDL particles. Small-dense
LDL (sdLDL) particles, the most atherogenic lipoproteins, are formed by the CETP-mediated exchange of cholesteryl ester (CE) and triacylglycerols (TAG) between VLDL and LDL particles. NASH (shown on the right side of the liver) could theoretically contribute to the low-grade inflammatory environment that is present in the atherosclerotic lesion. Finally, NAFLD (potentially steatosis, NASH or both) contributes to higher circulating PAI-1 levels, which impede the resolution of a thrombus. Other factors that may affect atherogenesis include insulin resistance-induced overproduction of glucose (which promotes monocyte/macrophage adhesion, SMC chemokine secretion and expression of an inflammatory phenotype in macrophages) and fetuin A secretion (which induces low-grade inflammation). This figure is available as part of a downloadable slideset 
events (PROactive) study showed a benefit for pioglitazone with respect to the secondary outcome (a composite of allcause mortality, non-fatal myocardial infarction and stroke) [37]. More recently, pioglitazone treatment in insulinresistant patients with recent ischaemic stroke or transient ischaemic attack (but without type 2 diabetes) resulted in a significant reduction in the occurrence of cardiovascular events [38].

Liraglutide has a similar effect on NASH (number needed to treat to resolve NASH after 48 weeks' treatment: 3) [39] and the Liraglutide Effect and Action in Diabetes: Evaluation of Cardiovascular Outcome Results (LEADER) trial showed that liraglutide was superior to standard care in reducing major adverse cardiovascular events in type 2 diabetes [40].

These interventions, however, do not specifically target NAFLD. It cannot, therefore, be concluded from these studies that NAFLD is causal in explaining CVD risk reduction.

MR studies More than 10 years ago, we were the first to show that the heritability of NAFLD, assessed by ultrasound, is 25$35 \%$ in dyslipidaemic pedigrees [41]. Subsequent linkage analyses revealed three quantitative trait loci on chromosome $1 \mathrm{q} 42.3,7 \mathrm{p} 12-21$ and 22p13-q11 that were associated with the fatty liver trait [41]. Of note, Romeo and colleagues later identified the gene encoding patatin-like phospholipase domaincontaining protein 3 (PNPLA3), located on chromosome 22q13.31, as the first NAFLD susceptibility gene [42], which has been associated with all stages of NAFLD [43].

The common variant in PNPLA3 (rs738409) was also used as an instrument in the first and at present only MR study to investigate the causal relationship between NAFLD and CAD. That study did not find any association [44]. In fact, we observed that the rs $738409 \mathrm{G}$ allele that predisposes to NAFLD conferred a modest protection from CAD in the CARDIoGRAMplusC4D dataset (www.cardiogramplusc4d. org; accessed 23 August 2019), consisting of 60,801 CAD cases and 123,504 controls [45]. This observation was confirmed in the Myocardial Infarction Genetics and CARDIoGRAM Exome Consortia study [46], which only partly overlaps with the CARDIoGRAMplusC4D dataset.

A similar protective effect has been found for the rs58542926 T allele (TM6SF2) a more-recently discovered NAFLD susceptibility gene encoding transmembrane 6 superfamily 2 (TM6SF2) [45, 47]. These apparently paradoxical observations may be explained by considering the functions of these two gene products. Although the true function of PNPLA3 remains to be elucidated, it has been suggested that it is involved in lipid droplet remodelling and VLDL production [48]. TM6SF2 is also involved in VLDL production (Fig. 3a) [49]. Indeed, variants in both PNPLA3 and TM6SF2 have also been associated with lower plasma lipid levels, both triacylglycerols and LDL-cholesterol [46], which might explain the negative relationship of these SNPs with CAD (Fig. 3b,c).
The simultaneous effects of PNPLA3 and TM6SF2 on both NAFLD and plasma lipids (through impaired VLDL production) are an example of horizontal pleiotropy. They are, therefore, not perfectly suited as instruments for MR studies, particularly when used in monogenic analyses (Text box 1). Furthermore, more recent studies have shown that the same variants in both PNPLA3 and TM6SF2 are also positively associated with type 2 diabetes $[46,50]$.

The third robust NAFLD susceptibility gene, GCKR (encoding liver-specific glucokinase regulatory protein [GKRP]), is involved in de novo lipogenesis (Fig. 3a) [51], one of the principal pathways in the development of NAFLD [2]. In a recent meta-analysis, we showed that common variants in this gene (rs1260326, rs780094 and rs780093, which are all in strong linkage disequilibrium) are modestly associated with CAD (OR per risk allele 1.02 [95\% CI 1.00, 1.04]) [52]. Of interest, these genetic variants have also been associated with higher serum triacylglycerols, lower serum HDLcholesterol and the presence of small-dense LDL particles [51], the lipid phenotype that characterises NAFLD [13]. Since it is believed that this lipid phenotype is a consequence of NAFLD (Fig. 3a) [51], it is an example of vertical pleiotropy (or mediation); the gene effect on lipids is through the liver, which does not invalidate the MR assumptions (Text box 1). It cannot, however, be ruled out that the common variants in GCKR also have horizontal pleiotropic effects. Previous studies have shown that these variants also protect against chronic kidney disease and type 2 diabetes [50,52].

Finally, variants in the membrane-bound $O$-acyltransferase domain-containing 7 gene (MBOAT7), which is involved in acyl-chain remodelling of phosphatidylinositols, have consistently been associated with NAFLD [53, 54]. Of interest, the rs641738 $\mathrm{T}$ allele was not associated with $\mathrm{CAD}$, nor with plasma lipids or type 2 diabetes $[45,50,55]$.

These studies suggest that plasma lipids play an important role in explaining the association between NAFLD and CAD (Fig. 3b,c). Moreover, given the opposing effects these NAFLD susceptibility genes have on type 2 diabetes risk (Fig. 3d), they also suggest that that plasma lipids have a greater impact on CAD risk than type 2 diabetes. Indeed, we recently expanded our genetic analyses to 12 NAFLD susceptibility genes (identified by either genome-wide association studies for NAFLD or NAFLD-related traits, or meta-analyses) and showed that the effects of these variants on CAD risk are largely accounted for by plasma lipids [55]. We observed a strong relationship between plasma lipids and CAD risk conferred by these NAFLD susceptibility genes [55]. Moreover, since many of these genes, including PNPLA3 and TM6SF2, have also been associated with NASH, it is questionable whether low-grade inflammation plays a major role in the connection between NAFLD and CVD. It should, however, be noted that PNPLA3 and TM6SF2 have not been associated with systemic low-grade inflammation $[56,57]$. 


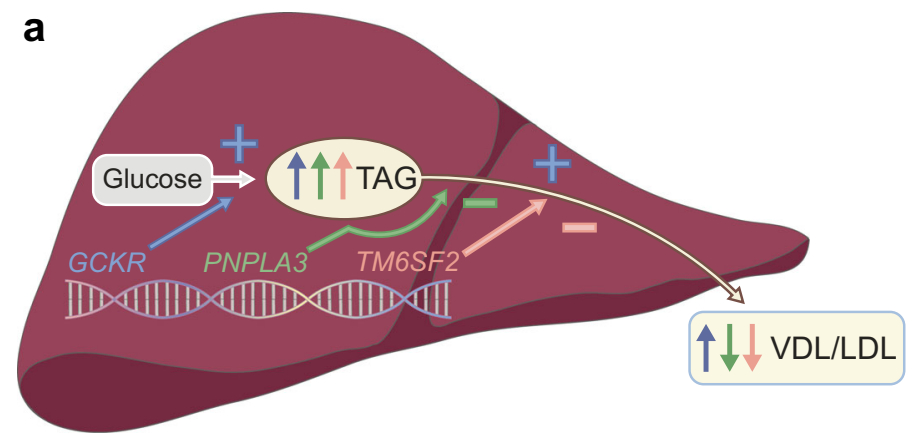

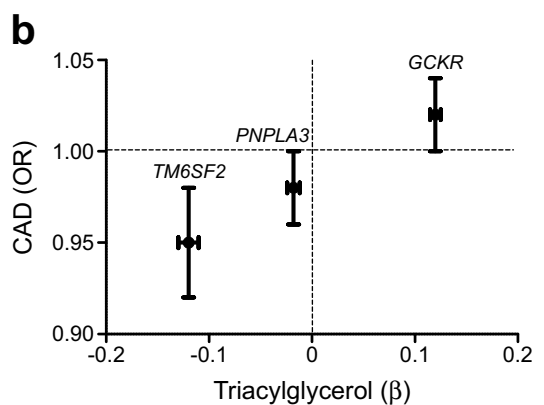

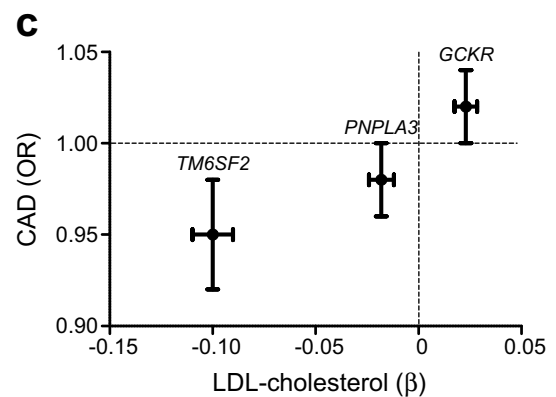

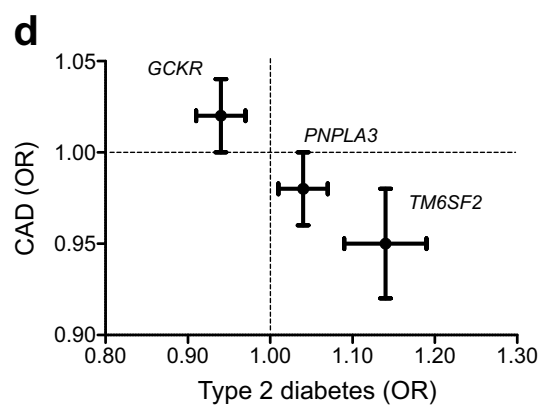

Fig. 3 Relationship of GCKR, PNPLA3 and TM6SF2 with plasma lipids, type 2 diabetes and CAD. (a) Variants in GCKR, PNPLA3 and TM6SF2 contribute to the development of intrahepatic triacylglycerol (TAG) accumulation by greater hepatic glucose uptake and de novo lipogenesis $(G C K R)$, impaired lipid-droplet remodelling (PNPLA3) and impaired VLDL secretion (PNPLA3 and TM6SF2). As a consequence, they have differential effects on plasma lipid levels. (b-d) Associations of common variants in GCKR, PNPLA3 and TM6SF2 with plasma triacylglycerols (b), LDL-cholesterol (c) and type 2 diabetes (d) (on $x$-axes) and CAD (on $y$-axes). Lipid data are derived from [46]; CAD data are derived from [45, 52]; type 2 diabetes data are derived from [50]. Error bars indicate $95 \%$ CIs. This figure is available as part of a downloadable slideset

\section{Clinical implications}

The high global prevalence of NAFLD has resulted in an exponential increase in the number and variety of drugs targeting steatosis, NASH and/or fibrosis that have entered Phase II and Phase III clinical trials [58]. Since these agents are aimed primarily at preventing progression to end-stage liver disease and hepatocellular carcinoma, it is important to underscore that the principal cause of death in individuals with NAFLD is CVD [5]. It is therefore essential that any antiNAFLD drug not only targets NAFLD but also has at least a neutral and preferably a protective effect on CVD events [58]. Given the intertwined relationship between NAFLD and plasma lipid levels (as indicated by the differential effects of NAFLD susceptibility genes on plasma lipids that determine CAD risk [55]), it is strongly recommended that plasma lipid levels are included as an important safety outcome measure in Phase II and Phase III clinical trials.

Another issue of concern is the development of drugs that may have NAFLD as a potential side effect. For instance, glucose-lowering drugs that act on hepatic glucokinase to increase hepatic glucose uptake (e.g. liver-specific glucokinase activators and disruptors of the GKRP-glucokinase complex $[59,60])$ could theoretically lead to an increased accumulation of hepatic fat via an increased de novo lipogenesis [61]. By using the common variant in GCKR as a model of life-long exposure to a modest increase in hepatic glucokinase activity (Fig. 3a), it can be predicted that it will indeed result in increased de novo lipogenesis and NAFLD, as well as dyslipidaemia and CVD [51, 52]. Of interest, we and others have shown that the effects of this common GCKR variant on hepatic fat accumulation and plasma triacylglycerols are more pronounced in conditions of obesity and hyperglycaemia [62, 63]. This would imply that obese individuals and those with poorly controlled type 2 diabetes are more prone to the undesired side effects of liver-specific glucokinase activators. Future studies are warranted to gain more insight into these potential side effects.

\section{Conclusions and future directions}

The MR approach can help to make causal inferences. Although this approach has its specific limitations (e.g. horizontal pleiotropy and statistical power), they can be overcome by combining large datasets using multiple SNPs. The first genetic studies in which NAFLD susceptibility genes were associated with CVD suggest that plasma lipids are an important mediator between both entities; this has important therapeutic consequences. A beneficial effect of a new drug for treating NAFLD may be offset by a greater CVD risk if the drug also increases plasma lipid levels. As hepatic fat 
accumulation drives the overproduction of lipoproteins, the histological stage 'simple steatosis' is not as simple (or benign) as the term suggests. Future studies should be aimed at unravelling the role of other NAFLD-mediated pathways, such as hepatic inflammation, in the pathogenesis of atherosclerosis.

Funding statement MCGJB is supported by a research grant from the Netherlands Heart Foundation (NHS; grant \#2015T042).

Duality of interest The authors declare that there is no duality of interest associated with this manuscript.

Contribution statement All authors were responsible for drafting the article and revising it critically for important intellectual content. All authors approved the version to be published.

Open Access This article is distributed under the terms of the Creative Commons Attribution 4.0 International License (http:// creativecommons.org/licenses/by/4.0/), which permits unrestricted use, distribution, and reproduction in any medium, provided you give appropriate credit to the original author(s) and the source, provide a link to the Creative Commons license, and indicate if changes were made.

\section{References}

1. Younossi ZM, Koenig AB, Abdelatif D, Fazel Y, Henry L, Wymer M (2016) Global epidemiology of nonalcoholic fatty liver disease: meta-analytic assessment of prevalence, incidence, and outcomes. Hepatology 64:73-84

2. Donnelly KL, Smith CI, Schwarzenberg SJ, Jessurun J, Boldt MD, Parks EJ (2005) Sources of fatty acids stored in liver and secreted via lipoproteins in patients with nonalcoholic fatty liver disease. $\mathrm{J}$ Clin Invest 115:1343-1351

3. Machado MV, Diehl AM (2016) Pathogenesis of nonalcoholic steatohepatitis. Gastroenterology 150:1769-1777

4. Charlton MR, Burns JM, Pedersen RA, Watt KD, Heimbach JK, Dierkhising RA (2011) Frequency and outcomes of liver transplantation for nonalcoholic steatohepatitis in the United States. Gastroenterology 141:1249-1253

5. Ong JP, Pitts A, Younossi ZM (2008) Increased overall mortality and liver-related mortality in non-alcoholic fatty liver disease. J Hepatol 49:608-612

6. Targher G, Byrne CD, Lonardo A, Zoppini G, Barbui C (2016) Non-alcoholic fatty liver disease and risk of incident cardiovascular disease: a meta-analysis. J Hepatol 65:589-600

7. Ekstedt M, Hagstrom H, Nasr P et al (2015) Fibrosis stage is the strongest predictor for disease-specific mortality in NAFLD after up to 33 years of follow-up. Hepatology 61:1547-1554

8. Santos RD, Valenti L, Romeo S (2019) Does nonalcoholic fatty liver disease cause cardiovascular disease? Current knowledge and gaps. Atherosclerosis 282:110-120

9. Targher G, Day CP, Bonora E (2010) Risk of cardiovascular disease in patients with nonalcoholic fatty liver disease. N Engl J Med 363: $1341-1350$

10. Stefan N, Haring HU, Cusi K (2019) Non-alcoholic fatty liver disease: causes, diagnosis, cardiometabolic consequences, and treatment strategies. Lancet Diabetes Endocrinol 7:313-324

11. Stefan N, Fritsche A, Schick F, Haring HU (2016) Phenotypes of prediabetes and stratification of cardiometabolic risk. Lancet Diabetes Endocrinol 4:789-798
12. Tabas I, Garcia-Cardena G, Owens GK (2015) Recent insights into the cellular biology of atherosclerosis. The Journal of cell biology 209:13-22

13. DeFilippis AP, Blaha MJ, Martin SS, et al. (2013) Nonalcoholic fatty liver disease and serum lipoproteins: the Multi-Ethnic Study of Atherosclerosis. Atherosclerosis 227: 429-436

14. Adiels M, Taskinen MR, Packard C et al (2006) Overproduction of large VLDL particles is driven by increased liver fat content in man. Diabetologia 49:755-765

15. Adiels M, Olofsson SO, Taskinen MR, Boren J (2008) Overproduction of very low-density lipoproteins is the hallmark of the dyslipidemia in the metabolic syndrome. Arterioscler Thromb Vasc Biol 28:1225-1236

16. Holmes MV, Ala-Korpela M, Smith GD (2017) Mendelian randomization in cardiometabolic disease: challenges in evaluating causality. Nat Rev Cardiol 14:577-590

17. Burgess S, Foley CN, Zuber V (2018) Inferring causal relationships between risk factors and outcomes from genome-wide association study data. Annu Rev Genomics Hum Genet 19:303-327

18. Do R, Willer CJ, Schmidt EM et al (2013) Common variants associated with plasma triglycerides and risk for coronary artery disease. Nat Genet 45:1345-1352

19. Voight BF, Peloso GM, Orho-Melander M et al (2012) Plasma HDL cholesterol and risk of myocardial infarction: a mendelian randomisation study. Lancet 380:572-580

20. Chapman MJ, Guerin M, Bruckert E (1998) Atherogenic, dense low-density lipoproteins. Pathophysiology and new therapeutic approaches. Eur Heart J 19(Suppl A):A24-A30

21. Targher G (2006) Relationship between high-sensitivity C-reactive protein levels and liver histology in subjects with non-alcoholic fatty liver disease. J Hepatol 45:879-881 author reply 881-872

22. Zacho J, Tybjaerg-Hansen A, Jensen JS, Grande P, Sillesen H, Nordestgaard BG (2008) Genetically elevated C-reactive protein and ischemic vascular disease. N Engl J Med 359:1897-1908

23. Ridker PM, Everett BM, Thuren T et al (2017) Antiinflammatory therapy with canakinumab for atherosclerotic disease. N Engl J Med 377:1119-1131

24. Mirea AM, Tack CJ, Chavakis T, Joosten LAB, Toonen EJM (2018) IL-1 family cytokine pathways underlying NAFLD: towards new treatment strategies. Trends Mol Med 24:458-471

25. Alessi MC, Bastelica D, Mavri A et al (2003) Plasma PAI-1 levels are more strongly related to liver steatosis than to adipose tissue accumulation. Arterioscler Thromb Vasc Biol 23:1262-1268

26. Song C, Burgess S, Eicher JD, O Donnell CJ, Johnson AD (2017) Causal effect of plasminogen activator inhibitor type 1 on coronary heart disease. J Am Heart Assoc 6:e04918

27. Mantovani A, Byrne CD, Bonora E, Targher G (2018) Nonalcoholic fatty liver disease and risk of incident type 2 diabetes: a meta-analysis. Diabetes Care 41:372-382

28. Korenblat KM, Fabbrini E, Mohammed BS, Klein S (2008) Liver, muscle, and adipose tissue insulin action is directly related to intrahepatic triglyceride content in obese subjects. Gastroenterology 134:1369-1375

29. Merino J, Leong A, Posner DC et al (2017) Genetically driven hyperglycemia increases risk of coronary artery disease separately from type 2 diabetes. Diabetes Care 40:687-693

30. Bornfeldt KE, Tabas I (2011) Insulin resistance, hyperglycemia. and atherosclerosis. Cell Metab 14:575-585

31. Stefan N, Haring HU (2013) The role of hepatokines in metabolism. Nat Rev Endocrinol 9:144-152

32. Weikert C, Stefan N, Schulze MB et al (2008) Plasma fetuin-a levels and the risk of myocardial infarction and ischemic stroke. Circulation 118:2555-2562

33. Fisher E, Stefan N, Saar K et al (2009) Association of AHSG gene polymorphisms with fetuin-A plasma levels and cardiovascular diseases in the EPIC-Potsdam study. Circ Cardiovasc Genet 2:607-613 
34. Laugsand LE, Ix JH, Bartz TM et al (2015) Fetuin-A and risk of coronary heart disease: a Mendelian randomization analysis and a pooled analysis of AHSG genetic variants in 7 prospective studies. Atherosclerosis 243:44-52

35. Pal D, Dasgupta S, Kundu R et al (2012) Fetuin-A acts as an endogenous ligand of TLR4 to promote lipid-induced insulin resistance. Nat Med 18:1279-1285

36. Cusi K, Orsak B, Bril F et al (2016) Long-Term pioglitazone treatment for patients with nonalcoholic steatohepatitis and prediabetes or type 2 diabetes mellitus: a randomized trial. Ann Intern Med 165:305-315

37. Dormandy JA, Charbonnel B, Eckland DJ et al (2005) Secondary prevention of macrovascular events in patients with type 2 diabetes in the PROactive Study (PROspective pioglitAzone Clinical Trial In macroVascular Events): a randomised controlled trial. Lancet 366:1279-1289

38. Kernan WN, Viscoli CM, Furie KL et al (2016) Pioglitazone after ischemic stroke or transient ischemic attack. N Engl J Med 374: 1321-1331

39. Armstrong MJ, Gaunt P, Aithal GP et al (2016) Liraglutide safety and efficacy in patients with non-alcoholic steatohepatitis (LEAN): a multicentre, double-blind, randomised, placebo-controlled phase 2 study. Lancet 387:679-690

40. Marso SP, Daniels GH, Brown-Frandsen K et al (2016) Liraglutide and cardiovascular outcomes in type 2 diabetes. N Engl J Med 375: 311-322

41. Brouwers MC, Cantor RM, Kono N et al (2006) Heritability and genetic loci of fatty liver in familial combined hyperlipidemia. $\mathrm{J}$ Lipid Res 47:2799-2807

42. Romeo S, Kozlitina J, Xing C et al (2008) Genetic variation in PNPLA3 confers susceptibility to nonalcoholic fatty liver disease. Nat Genet 40:1461-1465

43. Sookoian S, Pirola CJ (2011) Meta-analysis of the influence of I148M variant of patatin-like phospholipase domain containing 3 gene (PNPLA3) on the susceptibility and histological severity of nonalcoholic fatty liver disease. Hepatology 53:1883-1894

44. Lauridsen BK, Stender S, Kristensen TS et al (2018) Liver fat content, non-alcoholic fatty liver disease, and ischaemic heart disease: Mendelian randomization and meta-analysis of 279013 individuals. Eur Heart J 39:385-393

45. Simons N, Isaacs A, Koek GH, Kuc S, Schaper NC, Brouwers MC (2017) PNPLA3, TM6SF2, and MBOAT7 genotypes and coronary artery disease. Gastroenterology 152:912-913

46. Liu DJ, Peloso GM, Yu H et al (2017) Exome-wide association study of plasma lipids in $>300,000$ individuals. Nat Genet 49 : 1758-1766

47. Dongiovanni P, Petta S, Maglio C et al (2015) Transmembrane 6 superfamily member 2 gene variant disentangles nonalcoholic steatohepatitis from cardiovascular disease. Hepatology 61:506-514

48. Trepo E, Romeo S, Zucman-Rossi J, Nahon P (2016) PNPLA3 gene in liver diseases. J Hepatol 65:399-412

49. Kozlitina J, Smagris E, Stender S et al (2014) Exome-wide association study identifies a TM6SF2 variant that confers susceptibility to nonalcoholic fatty liver disease. Nat Genet 46:352-356
50. Dongiovanni P, Stender S, Pietrelli A et al (2018) Causal relationship of hepatic fat with liver damage and insulin resistance in nonalcoholic fatty liver. J Intern Med 283:356-370

51. Brouwers MC, Jacobs C, Bast A, Stehouwer CD, Schaper NC (2015) Modulation of glucokinase regulatory protein: a doubleedged sword? Trends Mol Med 21:583-594

52. Simons P, Simons N, Stehouwer CDA, Schalkwijk CG, Schaper NC, Brouwers M (2018) Association of common gene variants in glucokinase regulatory protein with cardiorenal disease: a systematic review and meta-analysis. PLoS One 13:e0206174

53. Mancina RM, Dongiovanni P, Petta S et al (2016) The MBOAT7TMC4 variant rs641738 increases risk of nonalcoholic fatty liver disease in individuals of European descent. Gastroenterology 150 e1216:1219-1230

54. Luukkonen PK, Zhou Y, Hyotylainen T et al (2016) The MBOAT7 variant rs641738 alters hepatic phosphatidylinositols and increases severity of non-alcoholic fatty liver disease in humans. J Hepatol 65:1263-1265

55. Brouwers MC, Simons N, Stehouwer CD, Koek GH, Schaper N, Isaacs A (2019) Relationship between nonalcoholic fatty liver disease susceptibility genes and coronary artery disease. Hepatol Commun 3:587-596

56. Kantartzis K, Peter A, Machicao F et al (2009) Dissociation between fatty liver and insulin resistance in humans carrying a variant of the patatin-like phospholipase 3 gene. Diabetes 58:2616-2623

57. Sookoian S, Castano GO, Scian R et al (2015) Genetic variation in transmembrane 6 superfamily member 2 and the risk of nonalcoholic fatty liver disease and histological disease severity. Hepatology 61:515-525

58. Francque S, Vonghia L (2019) Pharmacological treatment for nonalcoholic fatty liver disease. Adv Ther 36:1052-1074

59. Lloyd DJ, St Jean DJ Jr, Kurzeja RJ et al (2013) Antidiabetic effects of glucokinase regulatory protein small-molecule disruptors. Nature 504:437-440

60. Zhu D, Gan S, Liu Y et al (2018) Dorzagliatin monotherapy in Chinese patients with type 2 diabetes: a dose-ranging, randomised, double-blind, placebo-controlled, phase 2 study. Lancet Diabetes Endocrinol 6:627-636

61. Hodson L, Brouwers M (2018) Non-alcoholic fatty liver disease concerns with glucokinase activators. Lancet Diabetes Endocrinol 6:684-685

62. Simons N, Dekker JM, van Greevenbroek MM et al (2016) A common gene variant in glucokinase regulatory protein interacts with glucose metabolism on diabetic dyslipidemia: the combined CODAM and Hoorn Studies. Diabetes Care 39:1811-1817

63. Stender S, Kozlitina J, Nordestgaard BG, Tybjaerg-Hansen A, Hobbs HH, Cohen JC (2017) Adiposity amplifies the genetic risk of fatty liver disease conferred by multiple loci. Nat Genet 49:842847

Publisher's note Springer Nature remains neutral with regard to jurisdictional claims in published maps and institutional affiliations. 